\title{
Validation of internal control for gene expression study in soybean by quantitative real-time PCR
} Bo Jian ${ }^{1,2}$, Bin Liu ${ }^{2}$, Yurong Bi ${ }^{1}$, Wensheng $\mathrm{Hou}^{2}$, Cunxiang $\mathrm{Wu}^{2}$ and Tianfu Han*2

\author{
Address: ${ }^{1}$ School of Life Sciences, Lanzhou University, Lanzhou, Gansu 730000, PR China and ${ }^{2}$ The National Key Facility for Crop Gene Resources \\ and Genetic Improvement (NFCRI), Institute of Crop Sciences, The Chinese Academy of Agricultural Sciences, Beijing 100081, PR China \\ Email: Bo Jian - jianbo1007@yahoo.com; Bin Liu - bin.liu@bio.ntnu.no; Yurong Bi - yrbi@lzu.edu.cn; Wensheng Hou - houwsh@caas.net.cn; \\ CunxiangWu - wucx@mail.caas.net.cn; Tianfu Han* - hantf@mail.caas.net.cn \\ * Corresponding author
}

Published: 23 June 2008

BMC Molecular Biology 2008, 9:59 doi:10.1186/1471-2199-9-59
Received: 6 November 2007

Accepted: 23 June 2008

This article is available from: http://www.biomedcentral.com/I47/-2199/9/59

(c) 2008 Jian et al; licensee BioMed Central Ltd.

This is an Open Access article distributed under the terms of the Creative Commons Attribution License (http://creativecommons.org/licenses/by/2.0), which permits unrestricted use, distribution, and reproduction in any medium, provided the original work is properly cited.

\begin{abstract}
Background: Normalizing to housekeeping gene (HKG) can make results from quantitative realtime PCR (qRT-PCR) more reliable. Recent studies have shown that no single HKG is universal for all experiments. Thus, a suitable HKG should be selected before its use. Only a few studies on HKGs have been done in plants, and none in soybean, an economically important crop. Therefore, the present study was conducted to identify suitable HKG(s) for normalization of gene expression in soybean.
\end{abstract}

Results: All ten HKGs displayed a wide range of $\mathrm{Ct}$ values in $2 \mathrm{I}$ sample pools, confirming that they were variably expressed. GeNorm was used to determine the expression stability of the HGKs in seven series sets. For all the sample pools analyzed, the stability rank was ELFIB, CYP2 >ACTII > TUA > ELFIA > UBC2 > ACT2/7 > TUB > G6PD > UBQI0. For different tissues under the same developmental stage, the rank was ELFIB, CYP2 > ACT2/7 > UBC2 > TUA > ELFIA > ACTIII $>$ TUB $>G 6 P D>U B Q 10$. For the developmental stage series, the stability rank was ACT2/7, TUA > ELFIA $>U B C 2>E L F I B>T U B>C Y P 2>A C T I I>G 6 P D>U B Q 10$. For photoperiodic treatments, the rank was ACTII , ELFIB > CYP2 > TUA > ELFIA > UBC2 > ACT2/7 > TUB > G6PD > UBQI0. For different times of the day, the rank was ELFIA, TUA > ELFIB > G6PD > CYP2 > ACTII > ACT2/7 > TUB > $U B C 2>U B Q 10$. For different cultivars and leaves on different nodes of the main stem, the ten HKGs' stability did not differ significantly. $\Delta \mathrm{Ct}$ approach and 'Stability index' were also used to analyze the expression stability in all $2 \mathrm{I}$ sample pools. Results from $\triangle \mathrm{Ct}$ approach and geNorm indicated that ELFIB and CYP2 were the most stable HKGs, and UBQ 10 and G6PD the most variable ones. Results from 'Stability index' analysis were different, with ACTII and CYP2 being the most stable HKGs, and ELFIA and TUA the most variable ones.

Conclusion: Our data suggests that HKGs are expressed variably in soybean. Based on the results from geNorm and $\triangle \mathrm{Ct}$ analysis, ELFIB and CYP2 could be used as internal controls to normalize gene expression in soybean, while UBQ 10 and G6PD should be avoided. To achieve accurate results, some conditions may require more than one HKG to be used for normalization. 


\section{Background}

Gene expression analysis is becoming much more prevalent since it promotes our understanding of biological processes. Compared with the traditional methods for transcript analysis including Northern blot, RNase protection analysis, in situ hybridization and semi-RT-PCR, the fluorescence-based qRT-PCR has recently been considered as the most reliable method for the detection of mRNA [1] because of its high sensitivity, no post-PCR processing [2], and wide dynamic range [3], which allows a straightforward comparison between RNAs that differ widely in their abundance. Furthermore, it is easy to use, allows high throughput production of data and eliminates the need for radioactive isotopes [4]. Moreover, it is especially suitable when only a small number of cells are available. Although qRT-PCR is frequently used due to these advantages, some disadvantages may include variations between samples which may differ in the amount and quality of starting material, RNA preparation, cDNA synthesis, dilutions and pipetting[5]. Normalizing a target gene to the HKGs makes qRT-PCR reliable by minimizing the variations.

The HKGs, which are referred to as internal controls or reference genes, are presumed to have constant expression level among different tissues and at all developmental stages, regardless of the experimental conditions or treatments. Additionally, the HKG and target gene should have similar transcript levels to avoid analytical problems [6]. Commonly used HKGs are cellular maintenance genes, which regulate basic and ubiquitous cellular functions [7], such as components of the cytoskeleton (actins), glycolytic pathway (glyceraldehyde-3-phosphate dehydrogenase (GAPDH)), protein folding (cyclophilin), synthesis of ribosome subunits (rRNA), electron transporter (succinate dehydrogenase complex, SDH), protein degradation (ubiquitin), etc. These genes are supposed to have constant expression levels between different samples, and are frequently used as a normalizer without proper validation. However, recent studies show that the transcriptional levels of these HKGs are not always stable, and that no single HKG has a constant level under all experimental conditions [8-10]. A recent study even suggests that such a 'foolproof gene does not exist [11]. The reason for this expression variability may be that the HKGs not only take part in the basic cell metabolism but also participate in other cellular process $[12,13]$. Therefore, selecting a suitable HKG(s) which has a constant expression level in certain experimental conditions for normalization is crucial for getting accurate results in gene expression studies.

Recently, many procedures have been constructed to find the best suitable HKG(s) in a set of samples, such as geNorm [11], NormFinder [14], $\Delta \mathrm{Ct}$ approach [15] and 'Stability index' [16]. For example, using geNorm,
YWHAZ, GAPD and SDHA were found to be the most stable HKGs across the examined embryonic stages in bovine pre-implantation embryos, while the commonly used $A C T B$ was variably expressed [17]. By comparing the expression results of the non-stimulated tissues and leucocytes from Atlantic salmon (Salmo salar L.) using the Normfinder program, it was shown that EF1-alpha was the most stably expressed gene [18]. Using the $\Delta \mathrm{Ct}$ approach, GAPDH was found to be the most suitable HKG for expression studies in reticulocytes while the commonly used B2M should be avoided [15]. UBQ and TUA were selected as reference genes to normalize gene expression in a single female poplar hybrid clone $(P$. trichocarpa $\times P$. deltoidies) using the 'Stability index' [16].

Nevertheless, many studies on HKGs selection refer to human or animal tissue. As far as is known, only a few have been focused on plants such as rice [19-21], poplar [16], potato [22] and Arabidopsis thaliana [23]. Moreover, there is no report on soybean (Glycine max [L.] Merr.), a very important crop and a model plant in the early studies of photoperiodism [24,25].

Photoperiod controls several responses throughout the plant life cycle, such as germination [26], flowering induction [25], post-flowering development [27,28], maturity, dormancy [29], and yield formation [30,31]. The photoperiodic control of flowering in Arabidopsis, a long-day (LD) plant, is a hot topic attracting many scientists to enter this field and helping to better understand the processes involved [32-35]. However, there is little known about the mechanism in soybean, a typical short-day (SD) plant. Thus, the understanding of some key genes' expression patterns will help illuminate the mechanism involved in this process. Different cultivars of soybean may have different sensitivity to photoperiod; studies of the expression pattern of key genes in the photoperiodic pathway may also help elucidate what leads to the varietal difference.

In the present study, the expression profiles of ten HKGs, including ACT11, ACT2/7, TUA, TUB, UBC2, CYP2, G6PD, ELF1A, ELF1B and UBQ10, were studied during the development of Zigongdongdou (ZGGG), a late-maturing soybean (Glycine max [L.] Merr) cultivar, under LD and SD conditions. The expression patterns of the ten HKGs were also detected in soybean cultivar Heihe No. 27 (HH27), Zhonghuang No. 24 (ZH24) and Suinong No. 14 (SN14). GeNorm, $\Delta \mathrm{Ct}$ approach and 'Stability index' were used to assess the value of ten HKGs as suitable internal control(s) for soybean gene expression studies. 


\section{Results}

\section{Expression profiling of HKGs}

A qRT-PCR assay, based on SYBR Green detection, was designed for the transcriptional profiling of ten commonly used HKGs (ACT11, ACT2, G6PD, ELF1B, UBC2, ELF1A, TUB, TUA, CYP2 and UBQ10) in soybean. In order to select a reliable set of HKGs, all PCR assays were done in triplicate. To make the comparison among each PCR run reliable, all the $\mathrm{Ct}$ values were determined at the threshold fluorescence value of 0.2 , and three fixed PCR reactions were performed in every PCR run to make the data from each PCR run comparable. The $\mathrm{Ct}$ value was used to analyze the transcriptional levels of HKGs. This approach was a simplified way to give an overview of the abundance of the genes in the samples [36]. The ten HKGs showed a relatively wide range of $\mathrm{Ct}$ values from the lowest mean Ct value (18.22) in CYP2, to the highest (23.50) in ELF1A in all tested sample pools in soybean. Individual HKGs had different expression levels across all the sample pools tested. ACT11 and CYP2 showed the smallest gene expression variation (below 4 cycles), while ACT2/7, ELF1A, TUB and TUA had the highest expression variation (above 6 cycles) as shown in Figure 1. The wide expression range of the ten tested HKGs confirmed that no single HKG had a constant expression under these conditions in soybean. Obviously, it is necessary to select a suitable HKG to normalize gene expression under a certain condition.

\section{GeNorm analysis}

Gene expression stability $(\mathrm{M})$ of these ten HKGs in various tissue samples under different conditions was measured by geNorm as described by Vandesompele et al. [11]. This approach relies on the principle that the expression ratio of two ideal HKGs is constant in all the samples, independent of the experimental conditions and celltypes. Genes with the lowest $M$ have the most stable expression, while the highest $M$ value indicates the least stable expression [11]. We analyzed data under seven sets. As shown in Figure 2A, when all the 21 samples were taken together, the average expression stability value (M) of ELF1B and CYP2 was lowest, and that of UBQ10 was highest, suggesting that ELF1B and CYP2 had the most stable expression and that UBQ10 was expressed most variably. The results remained very similar in the different tissues under the same developmental stage series, with the lowest $M$ value for ELF1B and CYP2 (Figure 2B). $U B Q 10$ remained the least stable gene, while $A C T 2 / 7$ and TUA were the ones with the lowest $M$, indicating that they were stably expressed in the developmental series of soy-

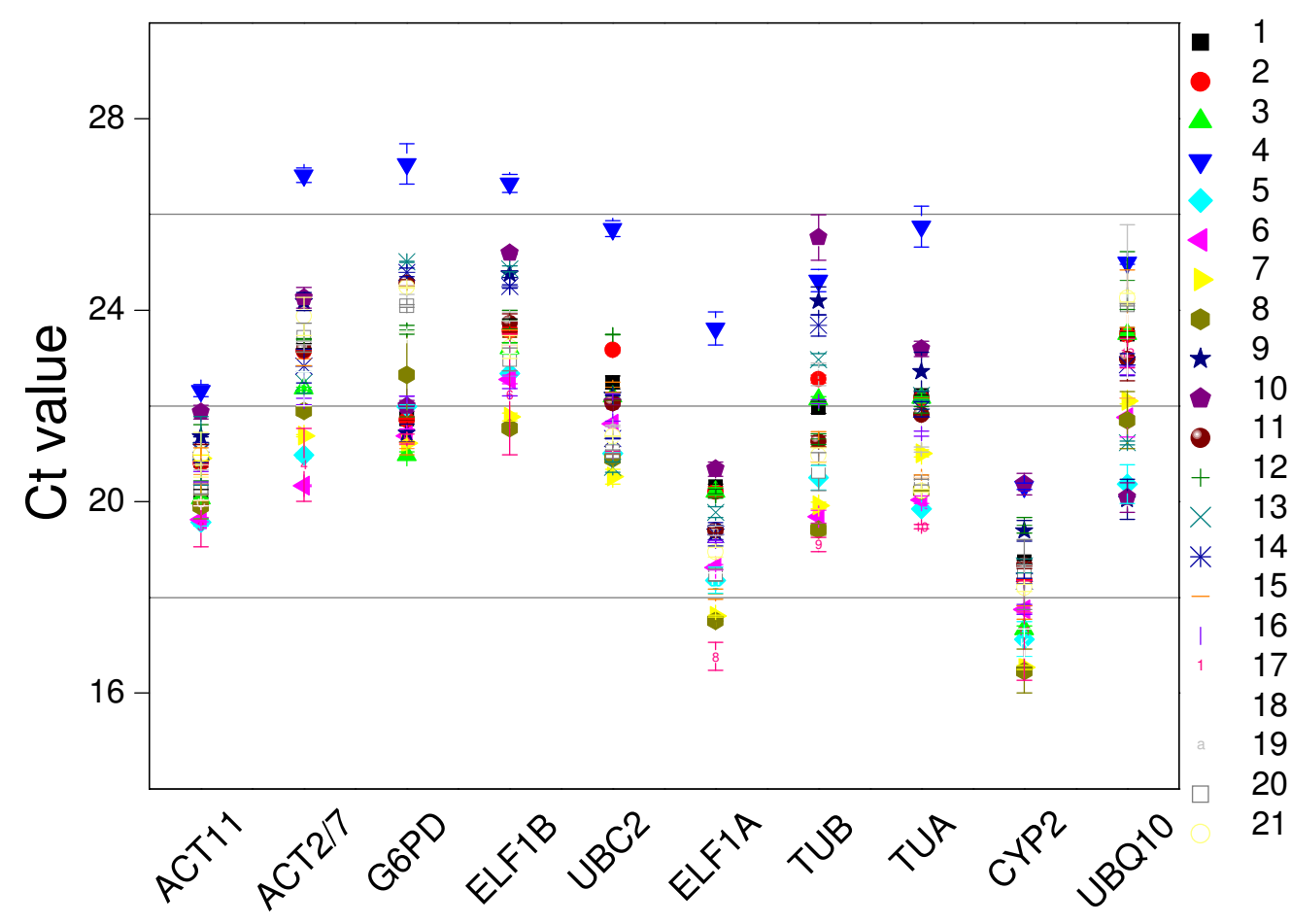

Figure I

The transcriptional profiles of individual HKGs in absolute $\mathbf{C t}$ values over all RNA samples. The tissues used for this analysis were listed in Table 4. The number indicated the corresponding sample. 

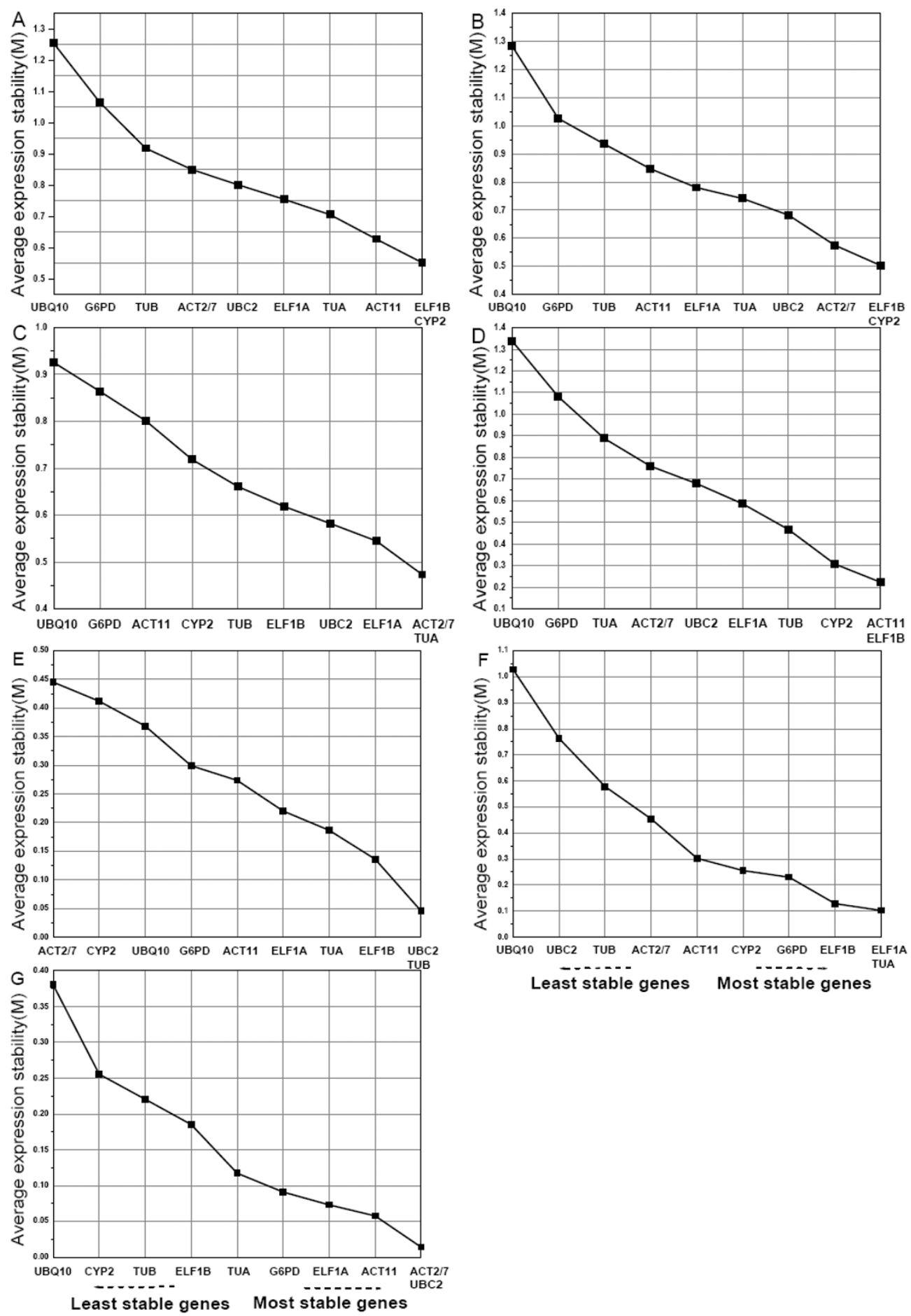

Least stable genes Most stable genes

\section{Figure 2}

Average expression stability and ranking of ten HKGs as calculated by geNorm. Expression stability and ranking of ten HKGs calculated with geNorm in all 21 sample pools (A), different tissues at the same developmental stage (B), developmental series $(C)$, photoperiod treatments $(D)$, different cultivar $(E)$, different time of the day series $(F)$, leaves located on different nodes on the main stem $(G)$. A lower average expression stability $M$ indicates more stable expression. 
bean (Figure 2C). In the photoperiodic treatment, ACT11 and $E L F 1 B$ were the most stable genes, while UBQ10 still was the most variable one (Figure 2D). In the different cultivar series, the $M$ value was least for $U B C 2$ and TUB followed by ELF1B, TUA, ELF1A, ACT11, while ACT2/7 was the least stable HKG (Figure $2 \mathrm{E}$ ). In the different time of the day series, ELF1A and TUA were expressed much more stably than the other eight HKGs, while UBQ10 continued to be the most variable one (Figure $2 \mathrm{~F}$ ). Since the HKGs UBQ10 and ACT11 showed variable expression profiles in a semi-RT-PCR comparison of the unifoliate leaves and a leaf mixture containing all the leaves (data not shown), the transcriptional expression of the ten HKGs was studied in this series. Results from geNorm analysis showed UBC2 and ACT2/7 were the least variable ones among the ten tested HKGs, while UBQ10 was still the most variable one. However, the difference of $M$ value between the less stable HKGs (ACT11, ELF1A, G6PD and TUA) was minimal (Figure $2 \mathrm{G}$ ).

For some experimental setups, using a single HKG for normalization is appropriate [20], while, for other ones, there may be no single HKG suitable as a reliable internal control [11]. Therefore, the requirement of two or more HKGs for accurate normalization is necessary. The optimal number of HKGs necessary for reliable normalization is defined by a normalization factor (NF) which is determined by the geNorm software. The pairwise variations $\mathrm{V}_{\mathrm{n}} / \mathrm{V}_{\mathrm{n}+1}$ between two sequential normalization factors $\left(\mathrm{NF}_{\mathrm{n}}\right.$ and $\left.\mathrm{NF}_{\mathrm{n}+1}\right)$ are used to determine the necessity of adding the next HKG for reliable normalization [11]. As shown in Figure 3(D,E,F,G), the two most stable HKGs were found to be optimal for the accurate normalization with a pairwise variation value much lower than the cutoff value of 0.15 suggested in [11]. It was apparent that the addition of the third HKG for normalization would have no significant effect on pairwise variation in the four series as shown in Figure 3A. When all 21 samples were taken together, the pairwise variation $\mathrm{V}_{2 / 3}$ was higher than 0.15 $(0.203)$, as was $V_{3 / 4}(0.174)$. This indicated the addition of the fourth HKG was necessary to normalize gene expression. This situation was similar for the series with different tissues under the same developmental stage. The pairwise variation $\mathrm{V}_{2 / 3}$ and $\mathrm{V}_{3 / 4}$ were 0.185 and 0.181 , respectively, both higher than 0.15 (Figure $3 \mathrm{~B}$ ). When looking at the developmental stages, the pairwise variation $\mathrm{V}_{2 / 3}$ was 0.177 , while $\mathrm{V}_{3 / 4}$ was 0.133 , so the three HKGs (ELF1A, ACT2/7 and TUA) were sufficient for accurate normalization. When evaluating all the pairwise variation, the least stable HKG was UBQ10 followed by G6PD as they significantly increased the pairwise variation during the whole assay by increasing the $\mathrm{V}$ value as shown in Figure 3.

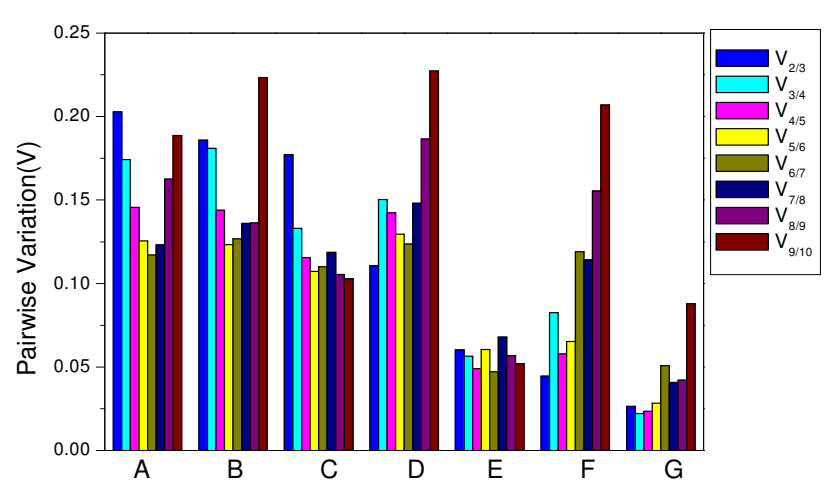

Figure 3

Determination of the optimal number of HKG for normalization by pairwise variation using geNorm. Pairwise variation (V) to determine the optimal number of HKG(s) for accurate normalization in all 2 I sample pools (A), different tissues at the same developmental stage (B), developmental series (C), photoperiod treatment (D), different cultivars $(E)$, different time of the day series $(F)$, leaves on different nodes on the main stem $(G)$.

\section{'Stability index' assay}

A 'Stability index' assay was first used to select suitable internal controls during the development of poplar [16]. In the current study, the expression stability rank of the ten HKGs in soybean was detected according to the 'Stability index'. As shown in Table 1, when all 21 sample pools were taken together, ACT11 had the lowest stability index and was the most stable HKG. The expression stability rank was as follows: ACT11 > CYP2 > UBC2 > ELF1B > ACT2/7 > G6PD > UBQ $10>$ TUB > TUA > ELF1A.

\section{$\Delta C$ t analysis}

The $\Delta \mathrm{Ct}$ approach was employed by Silver et al.[15] to select the most suitable HKG in reticulocytes. If the $\Delta \mathrm{Ct}$ value between two HKGs does not change when analyzed in all the samples, it means either both genes have stable expression patterns or they are co-regulated among those samples. However, the fluctuation in $\Delta \mathrm{Ct}$ means that at least one of them was variably expressed. Introduction of a third, fourth, or fifth gene into the comparisons will provide more information on which pairs show less variability and hence which gene(s) has stable expression among the samples tested. It is easy for large panels of genes to be compared with one another and then selected or eliminated on the basis of $\Delta \mathrm{Ct}$. Ultimately, an appropriate HKG can be selected for a particular experimental system [15].

The expression stability of HKGs was measured by the $\Delta \mathrm{Ct}$ value and standard deviation (StdDev) in the present study as described in [15]. Taking all ten HKGs into account and comparing all the possible combinations, their expression stability was determined. As shown in 
Table I: Summary of statistics measuring stability of HKG expression

\begin{tabular}{|c|c|c|c|c|c|c|}
\hline Gene & Mean $\mathrm{Ct}^{\mathrm{a}}$ & StdDevb & Slopec & Intercept $t^{d}$ & $\mathrm{CV} \% \mathrm{e}$ & Stability index \\
\hline ACTII & 18.63 & 0.74 & 0.03 & 20.97 & 3.57 & 0.11 \\
\hline CYP2 & 18.22 & 1.09 & 0.02 & 18.46 & 5.99 & 0.13 \\
\hline UBC2 & 21.91 & 1.17 & 0.03 & 21.91 & 5.33 & 0.16 \\
\hline ELFIB & 23.50 & 1.21 & 0.05 & 24.01 & 5.16 & 0.24 \\
\hline ACT2/7 & 22.82 & 1.42 & 0.05 & 23.33 & 6.21 & 0.28 \\
\hline G6PD & 22.91 & 1.68 & 0.04 & 22.45 & 7.34 & 0.31 \\
\hline$U B Q 10$ & 22.85 & 1.53 & 0.06 & 22.14 & 6.68 & 0.43 \\
\hline TUB & 21.77 & 1.74 & 0.09 & 22.80 & 7.99 & 0.72 \\
\hline TUA & 21.47 & 1.42 & 0.12 & 22.86 & 6.63 & 0.80 \\
\hline ELFIA & 19.25 & 1.43 & 0.12 & 20.58 & 7.45 & 0.90 \\
\hline
\end{tabular}

aData based on analysis of raw $\mathrm{Ct}$ value. Genes were ordered, top to bottom, from those tending to show the highest stability to those showing the lowest, based on the stability index. bStdDev among all the tissue samples tested. cSlope of regression of gene means. IIntercepts were also given for the estimated regression lines. eCoefficient of variation (CV) (StdDev divided by mean multiplied by 100). fStability index was the product of CV and slope (multiplication of columns 4 and 6 ). Genes with the lowest stability index usually provide the best control.

Additional File 1, Figure 4A and 4B, when ELF1B and CYP2 were compared with the other nine HKGs in their respective gene panels, the mean StdDev was 1.00 and 1.04 , respectively, indicating that $E L F 1 B$ was the most stable one among the ten HKGs analyzed in all the 21 sample pools, followed by CYP2. In contrast, when UBQ10 and G6PD were compared to the other nine HKGs in their respective gene panels, they tended to be associated with the greatest amount of deviation in $\Delta \mathrm{Ct}$ value (the mean StdDev was 1.84 and 1.50, respectively), which meant that $U B Q 10$, followed by G6PD, were the least stable HKGs in all the 21 samples tested. UBQ10 should be avoided when doing gene expression analysis because of its high deviation in $\Delta \mathrm{Ct}$ value when compared to the other nine HKGs (the greatest amount was 2.44 to TUB, and the least was 1.47 to UBC2). ELF1A, ACT11/TUA, TUB/ACT2/7 and UBC2 all showed intermediate levels of $\triangle \mathrm{Ct}$ deviation (the mean StdDev was 1.06, 1.07, 1.07, $1.08,1.08,1.15,1.50$ and 1.84 , respectively), indicating intermediate stability. Overall rankings were as follows: ELF1B, CYP2, ELF1A, ACT11/TUA, TUB/ACT2/7, UBC2, $G 6 P D$, and finally $U B Q 10$.

\section{GmBFT expression}

The relative expression level of GmBFT (Glycine max brother of FT and TFL1) [37], an ortholog of Arabidopsis $B F T$ [38], was detected to validate the HKGs selected in the present study under certain conditions according to the geNorm manual. The geometric average of the most stable HKGs (ACT2/7, TUA and ELF1A) in the developmental stage series selected by geNorm was used as an internal control. The relative expression of GmBFT increased after a 25-day SD treatment in unifoliate leaves, being 5.5-fold higher than that at 1-day SD treatment (Figure $5 \mathrm{~A} \mathrm{~b}$ and a, Table $2 \mathrm{~b}$ and a). Similarly, GmBFT expression level was also higher in shoot tips after a 25-day SD treatment compared to the 1 -day SD treatment (Figure 5A $\mathrm{d}$ and $\mathrm{c}$, Table $2 \mathrm{~d}$ and $\mathrm{c}$ ). In the same way, using the geometric average of the most stable HKGs (ACT11 and ELFIB) in the photoperiodic treatment selected by geNorm as an internal control, it was found that the relative expression of GmBFT expression in both unifoliate leaves and shoot tips at 25-day under SD treatment was higher than that under LD treatment. The expression level of GmBFT in unifoliate leaves under 25-day SD treatment was 2.98 fold higher than that under 25-day LD treatment (Figure 5B b and e, Table $3 \mathrm{a}$ and $\mathrm{b}$ ). Likewise, GmBFT expression level in shoot tips under 25-day SD treatment was 25.3 fold higher than that under 25-day LD treatment (Figure 5B d and f, Table $3 \mathrm{c}$ and $\mathrm{d}$ ). As shown previously by geNorm in the present study, GAPD and UBQ10 were the most variable HKGs for the developmental stage and photoperiodic treatment series. The relative expression of GmBFT in unifoliate leaves and shoot tips was also detected using the two HKGs as internal controls and no significant difference was found.

\section{Discussion}

qRT-PCR has become a powerful tool for gene expression analysis because of its high throughput, sensitivity and accuracy $[1,4]$. The use of suitable HKGs to normalize the variation made by RNA preparation, cDNA synthesis or PCR processing would make the results more reliable. In order to select suitable HKG(s) for normalization, many procedures such as geNorm [11], Normfinder [14], $\Delta \mathrm{Ct}$ approach [15] and 'Stability index' [16] have been used. Since all methods mentioned above are based on the $\mathrm{Ct}$ value, which is determined mostly by the quantity of cDNA [39], the prerequisite for selecting a set of reliable HKG(s) is based on the equal input cDNA when doing qRT-PCR. In early studies, the most commonly used method to measure the input cDNA was by a spectrophotometer such as NanoDrop ND-1000 [20,40,41]. Considering the importance of input CDNA, more than one 

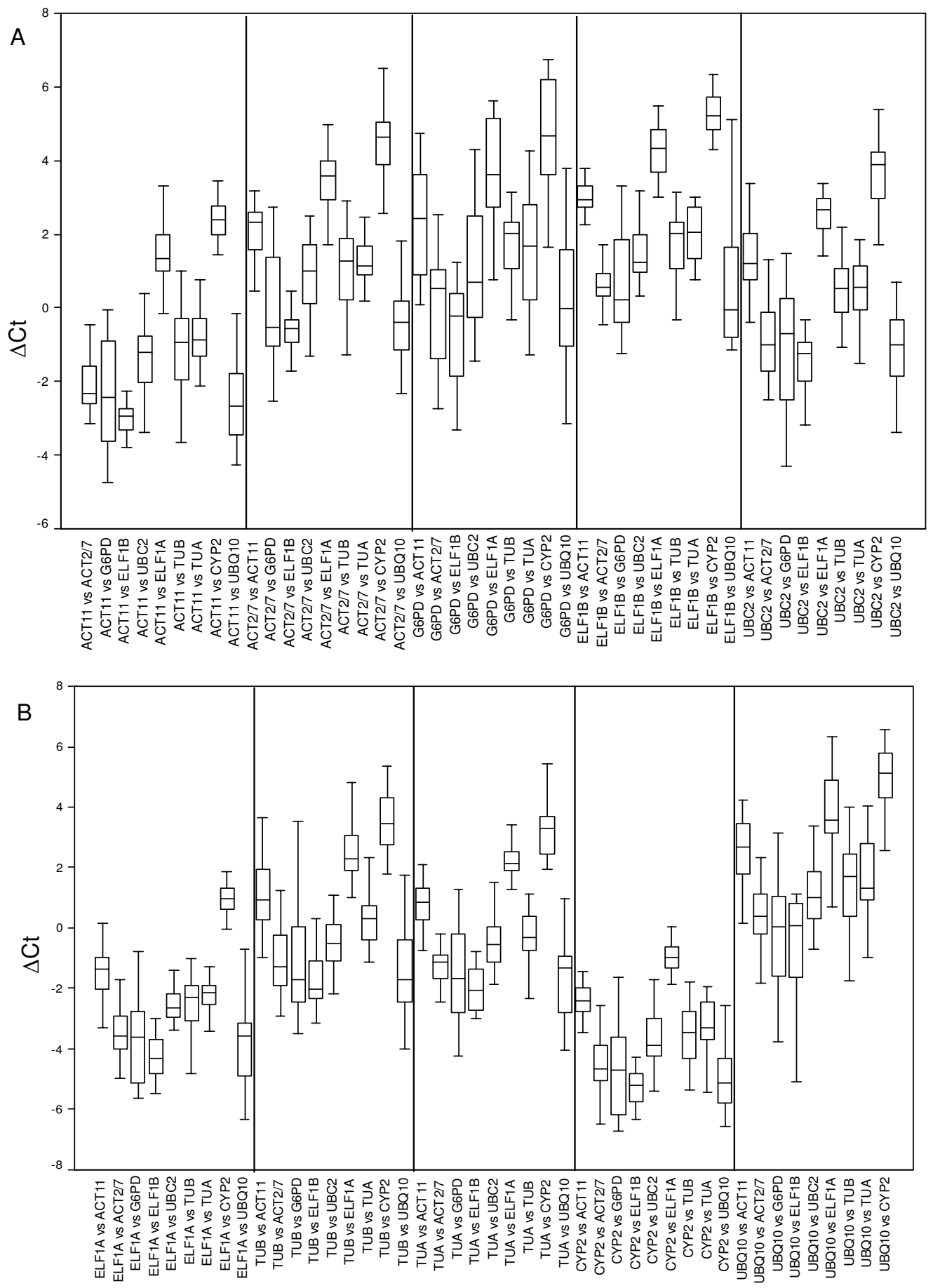

Gene comparisons

\section{Figure 4}

$\Delta \mathbf{C t}$ method for HKG selection. $\Delta \mathrm{Ct}$ variability in HKG comparisons were shown as medians (lines), 25th percentile to the 75th percentile (boxes) and ranges (whiskers) for all the 21 sample pools. A. Comparisons of the completely possible sets of HKGs which included ACTI I, ACT2/7, G6PD, ELFIB, UBC2; B. Comparisons of the completely possible sets of HKGs, which included ELFIA, TUB, TUA, CYP2 and UBQIO. 

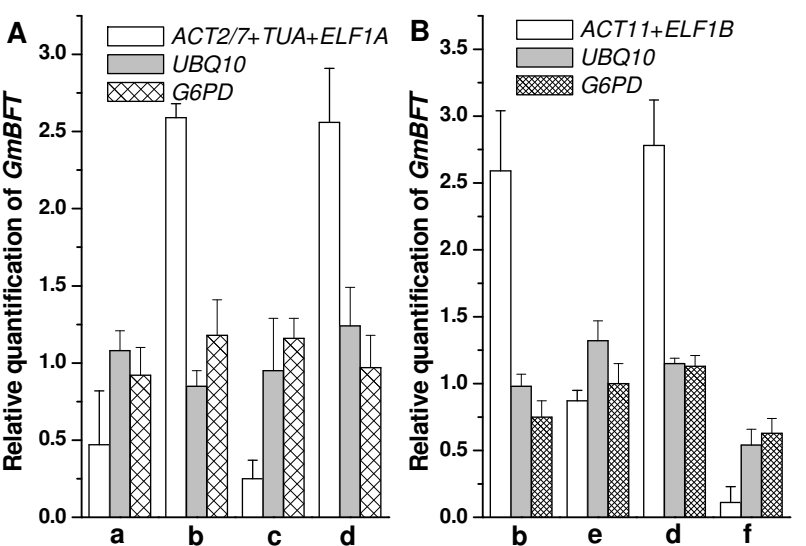

Figure 5

Relative quantification of GmBFT expression using different HKGs as internal controls under different developmental stages and photoperiod conditions. Relative quantification of GmBFT expression was detected using two or three of the most stable HKGs or the most variable HKGs selected by geNorm as internal controls. The geometric average of ACT2/7, TUA and ELFIA, UBQ 10 and G6PD were used as internal controls for developmental stage (A), the geometric average of $A C T / I$ and ELFIB, UBQ IO and G6PD were used as internal controls for photoperiod treatment (B). a, SD I-day leaves; b, SD 25-day leaves; c, SD I-day shoot tips; d, SD 25- day shoot tips; e, LD 25-day leaves; f, LD 25-day shoot tips.

method should be used to detect its quantity and quality to ensure the cDNA equality for each PCR run in order to get reliable results for $\mathrm{HKG}(\mathrm{s})$ expression stability analysis. In the present study, cDNA was verified by measurements on both the ND-1000 and SMA3000 spectrophotometers to ensure the equality of CDNA in the PCR reactions and reliability of the results when using $\mathrm{Ct}$ values for analysis.

When gene expression stability in soybean was analyzed by geNorm, the most stable genes in the seven series were different as shown in Figure 3. In all seven series analyzed, ELF1B was the most stable HKG. UBQ10 and G6PD were the most variable ones, so these genes should be avoided as internal controls when doing gene expression studies in soybean. Our findings were in accordance with the result that UBQ10 exhibited the least stable expression in different tissues or cell types at different developmental stages in rice [20]. Similarly, in the development of grape berry, UBQ10 was not the recommended HKG for normalization [42]. However, in an earlier study in Arabidopsis, UBQ10 showed highly stable expression [23]. An ubiquitin tag is not only used to mark particular proteins for proteolytic elimination, but can also have non-proteolytic functions [43] which may lead to the variable expression of ubiquitin in different plants. G6PD was suggested to be an inappropriate internal control in qRT-PCR studies of estrogens effects in fish [44] and it also showed significant differences in expression between malignant and nonmalignant pairs (at least $\mathrm{p}<0.04$ ) of human bladder cancer [45]. There are few published works using G6PD as an internal control. This may be because G6PD not only acts as a component of the glycolytic pathway but also participates in other processes as well. Thus, the expression profile of G6PD might change according to the corresponding experimental conditions. In the present study, TUA was found to be one of the most stable HKGs with the lowest $M$ value in the developmental series in soybean. This result was consistent with an earlier study in poplar with TUA as one of the most stable HKGs [16]. Other most commonly used HKGs, like TUB, displayed an unacceptably high variable expression pattern limiting its use as an internal control except in the different cultivar series where all the other HKGs showed relatively stable expression. Taken together, these results suggested that these HKGs were regulated differently in each plant species and may exhibit differential expression patterns. Therefore, a HKG with stable expression in one organism may be not suitable to normalize gene expression in another organism under a given set of conditions and thus needs to be validated before its use.

To further verify the suitability of HKGs selected in the present study, GmBFT expression levels were detected at different developmental stages and under different photoperiodic conditions in soybean (Figure 5). In unifoliate leaves, GmBFT expression after a 25-day SD treatment was

Table 2: Relative expression of GmBFT at different developmental stages

\begin{tabular}{|c|c|c|c|}
\hline \multirow[t]{2}{*}{ Tissue sample } & \multicolumn{3}{|c|}{ Relative expression of GmBFT using different HKG(s) as internal controls (mean \pm StdDev) } \\
\hline & $A C T 2 / 7+T U A+E L F I A$ & UBQIO & G6PD \\
\hline a & $0.47 \pm 0.35$ & $1.08 \pm 0.13$ & $0.92 \pm 0.18$ \\
\hline $\mathrm{b}$ & $2.59 \pm 0.09$ & $0.85 \pm 0.10$ & $1.18 \pm 0.23$ \\
\hline c & $0.25 \pm 0.12$ & $0.95 \pm 0.34$ & $1.16 \pm 0.13$ \\
\hline d & $2.56 \pm 0.35$ & $1.24 \pm 0.25$ & $0.97 \pm 0.21$ \\
\hline
\end{tabular}

a, SD I-day leaves; b, SD 25-day leaves; c, SD I-day shoot tips; d, SD 25- day shoot. 
Table 3: Relative expression of GmBFT under different photoperiodic treatments

\begin{tabular}{|c|c|c|c|}
\hline \multirow[t]{2}{*}{ Tissue sample } & \multicolumn{3}{|c|}{ Relative expression of GmBFT using different $\mathrm{HKG}(\mathrm{s})$ as internal controls (mean \pm StdDev) } \\
\hline & $A C T I I+E L F I B$ & UBQIO & G6PD \\
\hline a & $2.59 \pm 0.45$ & $0.98 \pm 0.09$ & $0.75 \pm 0.12$ \\
\hline$b$ & $0.87 \pm 0.08$ & $1.32 \pm 0.15$ & $1.00 \pm 0.15$ \\
\hline c & $2.78 \pm 0.34$ & $1.15 \pm 0.04$ & $1.13 \pm 0.08$ \\
\hline d & $0.11 \pm 0.12$ & $0.54 \pm 0.12$ & $0.63 \pm 0.11$ \\
\hline
\end{tabular}

a, SD 25-day leaves; b, LD 25-day leaves; c, SD 25- day shoot tips; d, LD 25-day shoot tips.

significantly higher than after a 1-day SD treatment, and was also higher than after a 25-day LD treatment. The case was similar in the shoot tips of soybean using HKGs selected in corresponding conditions as internal controls. The fact that this result is in accordance with earlier work by Sun et al. [37] means that the HKGs identified in this study are suitable at various development stages and photoperiodic conditions. The relative expression of GmBFT was also analyzed using $U B Q 10$ and G6PD as internal controls (Figure 5). No significant expression difference of GmBFT was observed in unifoliate leaves at different developmental stages or under different photoperiodic conditions. The result was similar in shoot tips. Obviously, UBQ10 and G6PD are not suitable HKGs to normalize gene expression in soybean under such conditions.

The $\Delta \mathrm{Ct}$ approach [15] and 'Stability index' [16] were also used to analyze gene expression stability in all 21 sample pools in soybean to compare the accuracy of these three methods. Results obtained from the $\Delta \mathrm{Ct}$ method were very similar to that from the geNorm analysis. ELF1B was the most stable HKG followed by CYP2, while UBQ10 was the most variable HKG followed by G6PD. When 'Stability index' was used to measure the stability of HKG(s), results changed, with ACT11 being the most stable HKG followed by CYP2, while TUA and ELF1A became the least stable ones in all the 21 sample pools in soybean. An explanation might be that the 'Stability index'did not take the PCR efficiency into account, which played an important role in the data analysis. Thus, in order to get a reliable result by only comparing $\mathrm{Ct}$ deviation for the individual genes in the tested tissue samples, the HKGs analyzed should have similar PCR efficiency.

Although gene expression stability analyzed by the $\Delta \mathrm{Ct}$ approach was similar to that by geNorm, it was still not the first choice to get accurate normalization especially for the research which can get enough samples. The $\Delta \mathrm{Ct}$ method can be used to detect the most stable HKG as geNorm provides; however, it could not provide the number of HKGs necessary for accurate normalization. Some studies may require more than one HKG to be included, and using the single most stable HKG for nor- malization might not get the most accurate result. Indeed, as shown in Figure 3A, when all 21 sample pools were analyzed by geNorm, the geometric average of four HKGs (ELF1B, CYP2, ACT11 and TUA) was recommended as the normalization factor to get accurate results in soybean. However, the analysis by $\Delta \mathrm{Ct}$ method only indicated that the most stable HKG was ELF1B, and it could not be used to find out the optimal number of HKGs. Thus, gene expression accuracy might be undermined if the result is only based on ELF1B. It is true that the $\triangle \mathrm{Ct}$ method is useful for validating the most stable HKG in some specific tissue samples or cell types for which it is difficult to obtain enough material, such as reticulocytes [15]. However, for material where RNA samples are easy to obtain, such as plants, geNorm is recommended because it is easy to determine the optimal number of stable HKGs for accurate normalization.

Selection of suitable HKG(s) is necessary for accurate gene expression, but it is quite expensive and time-consuming. To avoid the additional expense and labor of using multiple internal control genes, a potential strategy suggested by Brunner et al. [16] was to design a PCR primer pair which could amplify two or more members of a control gene family. However, for two members of the same HKG family, their expression pattern and stability might vary. Therefore, results may be questionable for gene expression based on these two HKGs. For example, UBQ5 was one of the most suitable HKGs in a given set of tissue samples in rice, while UBQ10 expressed variably [20]. A similar situation was observed for the actin gene family in the developmental stage series in the present study. ACT2/7 was stably expressed, while ACT11 showed variable profiling. Thus, using primer pair sets to amplify two or more members of a HKG family for qRT-PCR may not be recommendable. After all, the accuracy of the results should always be given first priority.

The photoperiod plays an important role throughout the life cycle of soybean $[27,28,46]$. The sensitivity of different cultivars of soybean to photoperiod is quite variable. Understanding the mechanisms involved in this process will be beneficial in the molecular breeding of soybean. In 
the present study, the most stable HKG in the different cultivar series under the same photoperiodic conditions and developmental stage was $U B C 2$, whereas $A C T 2 / 7$ was the most variable one. The difference of average expression stability among the ten HKGs in this series was smaller than that in the other series. Thus, the gene expression would be similar using any of the ten HKGs as internal controls when studying different cultivar. It would be helpful to understand the mechanisms involved in differential sensitivities to photoperiod for different cultivars.

\section{Conclusion}

A large number of studies have been carried out concerning the validation of HKG(s) in many different tissue samples and cell types. However, there is no correlative report in soybean, a SD dicot. Our data showed the variable expression profiles of ten commonly used HKGs in different tissue samples and under different photoperiodic conditions in soybean. Based on geNorm and $\Delta \mathrm{Ct}$ methods, $E L F 1 B$ and CYP2 appears to be the most suitable HKGs to normalize gene expression during the development of soybean, while UBQ10 and G6PD seems to be unsuitable as reference genes. Under some conditions, more than one HKG should be used as internal controls to normalize gene expression in soybean in order to get the most reliable results.

\section{Methods}

\section{Sample collection and RNA extraction}

ZGGG, a soybean (Glycine max [L.] Merr.) cultivar from Sichuan Province, South China, was used as the main material. This cultivar is late maturing and sensitive to photoperiod $[27,28]$. HH27, a soybean cultivar from Heilongjiang Province, which is not sensitive to photoperiod; ZH24, a soybean cultivar from Beijing and SN14, another soybean cultivar from Heilongjiang, were also used as the materials. After the unifoliate leaves expanded, the seedlings were transferred to LD ( $16 \mathrm{~h}$ light $/ 8 \mathrm{~h}$ dark) and SD (12 h light/12 h dark) conditions, respectively [28]. The samples were collected and then frozen in liquid $\mathrm{N}_{2}$ and stored at $-80^{\circ} \mathrm{C}$ until RNA extraction. All the sample pools used for this research were provided in Table 4 and each pool contained at least 30 seedlings.

Total RNA was extracted using Trizol (Invitrogen) according to the Manufacture's Instruction with little modification. One more chloroform extraction step was added to the RNA extraction process. RNA was quantified by the absorbance at $\mathrm{OD}_{260}$ using NanDrop ND-1000 spectrophotometer. The absorbance ratio at $\mathrm{OD}_{260 / 280}$ and $\mathrm{OD}_{260 / 230}$ were used to assess the purity of all the RNA samples. Only RNA samples with $\mathrm{OD}_{260 / 280}$ ratio (protein contamination) between 1.8-2.0 and $\mathrm{OD}_{260 / 230}$ (organic pollutant) higher than 2.0 was used for the further analysis. RNA integrity was verified by $2 \%$ agar gel electrophoresis and ethidium bromide staining. The samples with 25S/18S ribosomal RNA between 1.5-2.0 and absence of smears were used for the following experiment.

cDNA synthesis and quantification

Before cDNA synthesis, $5 \mu \mathrm{g}$ total RNA was treated with RQ1 RNase-free DNase (Promega) according to the Man-

Table 4: Soybean tissues used for gene expression analysis

\begin{tabular}{ccccc}
\hline No. & Cultivar & Organ & Photoperiod & Time after photoperiodic treatment for seeding \\
\hline I & ZGDD & Root & LD & Id \\
2 & ZGDD & Root & LD & 25d \\
3 & ZGDD & Root & SD & Id \\
4 & ZGDD & Stem & SD & 25d \\
5 & ZGDD & Stem & SD & 25d \\
6 & ZGDD & Stem & LD & 25d \\
7 & ZGDD & Shoot tip & SD & Id \\
8 & ZGDD & Shoot tip & SD & $25 \mathrm{~d}$ \\
9 & ZGDD & Root nodule & SD & $25 \mathrm{~d}$ \\
10 & ZGDD & Root nodule & LD & $25 \mathrm{~d}$ \\
11 & ZGDD & Leaf & SD & $25 \mathrm{~d}$ \\
12 & ZGDD & Leaf & LD & $2 \mathrm{~h}$ \\
13 & ZGDD & Leaf & LD & $6 \mathrm{~h}$ \\
14 & ZGDD & Leaf & LD & IO h \\
15 & ZGDD & Leaf & LD & 25d \\
16 & ZGDD & Flower & SD & $32 \mathrm{~d}$ \\
17 & ZGDD & Pod & SD & Id \\
18 & ZGDD & Leaf & LD & Id \\
19 & HH27 & Leaf & LD & Id \\
20 & ZH24 & Leaf & LD & Id \\
21 & SNI4 & Leaf & LD &
\end{tabular}


ufacture's Instruction to ensure no DNA contamination and then cDNA synthesis was carried out with the purified RNA using the SuperScript III First-Strand Synthesis System (Invitrogen) following the instruction. The RT reaction was performed using Mastercycler Gradient (Eppendorf). Briefly, $1 \mu \mathrm{g}$ RNA, $50 \mu \mathrm{M}_{\text {oligo }_{\mathrm{dT}}}(20)$ and 10 $\mathrm{mM}$ dNTP mix were added together to incubate at $65^{\circ} \mathrm{C}$ for $5 \mathrm{~min}$, then placed on ice for at least $1 \mathrm{~min}$. After that, $2 \mu \mathrm{l} 10 \times$ RT buffer, $1 \mu \mathrm{l} 25 \mathrm{mM} \mathrm{MgCl} 2,2 \mu \mathrm{l} 0.1 \mathrm{M}$ DTT, 40 U RNaseOUT and 200 U SuperScript III were added and then incubated at $50^{\circ} \mathrm{C}$ for $50 \mathrm{~min}$. The RT reaction was terminated by incubating at $85^{\circ} \mathrm{C}$ for $5 \mathrm{~min}$ and the residual RNA was removed by incubated at $37^{\circ} \mathrm{C}$ for 20 min with the addition of $1 \mu \mathrm{l}$ RNaseH. After CDNA was synthesized, it was used as the template for PCR amplification. This amplification was made using primer pair sets which span an intron to detect DNA contamination.

cDNA was $2 \times$ diluted before quantification and the quantity and quality of input cDNA were determined by the SMA3000 and NanDrop ND-1000 spectrophotometer to make sure the cDNA amount for each PCR run is equal. For each method, the measurement was done in duplicate. The slope of the regression line of the concentrations measured with both methods did not differ indicating the equality of cDNA measurement with both methods for the qRT-PCR reaction (Yconc.ND-1000 $=1.0444 \times$ Xconc.SMA3000+0.5443; $\mathrm{n}=21 ; \mathrm{r}=0.9763$ ). To reduce the system error, all the cDNA was diluted to about 2.3 $\mathrm{ng} / \mu \mathrm{l}$, so there were $20 \mathrm{ng} / 8.8 \mu \mathrm{l} \mathrm{cDNA}$ for the real-time
RT-PCR reaction. All the CDNA were stored at $-20^{\circ} \mathrm{C}$ until PCR.

\section{Selection of soybean sequences, primer design and PCR optimization}

Sequences for the primer design were selected according to Brunner et al. [16] to identify soybean homologs for genes which are commonly used as internal controls. The soybean EST database [47] was queried with the relevant Arabidopsis protein using TBLASTN. Selected soybean ESTs were then used to query the Arabidopsis protein database using BLASTX. Primers were designed with Primer Premier 5 [48] with melting temperature between $60-62^{\circ} \mathrm{C}$, $18-20 \mathrm{bp}$ and about $50 \%$ GC content. The primers were used to query soybean EST database with BLASTN to ensure the specificity for the selected gene family member (Table 5). Since there is little known about the genomic DNA sequence of soybean, alignments were made with DNA sequence of relevant orthologs in Arabidopsis before primer design to ensure the primer pairs span at least one intron. The primer sequence, primer positions (indicating that the primers span an intron) and amplicon length were provided in Table 6 . Before qRT-PCR, the primer pairs were tested by standard PCR reaction with Mastercycler Gradient (Eppendorf) to find out the best suitable conditions. Amplicons of expected size were verified by $2 \%$ agarose gel electrophoresis and ethidium bromide staining.

Table 5: Description of soybean genes for qRT-PCR

\begin{tabular}{|c|c|c|c|c|c|}
\hline $\mathrm{Name}^{\mathrm{a}}$ & $\begin{array}{c}\text { Soybase Accession } \\
\text { Number }\end{array}$ & $\begin{array}{l}\text { Arabidopsis homolog } \\
\text { locus }^{\mathrm{b}}\end{array}$ & $\begin{array}{l}\text { Arabidopsis locus } \\
\text { description }\end{array}$ & Function & BLASTX score/E Value \\
\hline ACTII & TC204I37 & AT3G 12110 & Actin I I & $\begin{array}{c}\text { Cytoskeletal structural } \\
\text { protein }\end{array}$ & $740 / 0$ \\
\hline ACT2/7 & TC204I50 & AT5G098I0 & Actin $2 / 7$ & $\begin{array}{c}\text { Cytoskeletal structural } \\
\text { protein }\end{array}$ & $739 / 0$ \\
\hline G6PD & TC224599 & AT5G40760 & $\begin{array}{l}\text { Glucose-6-phosphate } \\
\text { dehydrogenase }\end{array}$ & Glucose metabolic process & $903 / 0$ \\
\hline ELFIB & TC203623 & AT5GI95I0 & $\begin{array}{l}\text { Eukaryotic elongation } \\
\text { factor I-beta }\end{array}$ & Translational elongation & $245 / 2 e-65$ \\
\hline$U B C 2$ & TC2I 4734 & AT2G02760 & $\begin{array}{l}\text { Ubiquitin-conjugating } \\
\text { enzyme E2 }\end{array}$ & $\begin{array}{l}\text { Ubiquitin-dependent } \\
\text { protein catabolic process }\end{array}$ & $314 / 5 e-86$ \\
\hline ELFIA & TC203954 & AT5G60390 & $\begin{array}{l}\text { Eukaryotic elongation } \\
\text { factor I-alpha }\end{array}$ & Translational elongation & $469 / e-132$ \\
\hline TUB & TC203804 & ATIG500I0 & Beta-tubulin & $\begin{array}{l}\text { Structural constituent of } \\
\text { cytoskeleton }\end{array}$ & $847 / 0$ \\
\hline TUA & AY907702 & AT5GI9780 & Tubulin alpha-5 & $\begin{array}{l}\text { Structural constituent of } \\
\text { cytoskeleton }\end{array}$ & $803 / 0$ \\
\hline CYP2 & TC224926 & AT2G21130 & Cyclophilin & Protein folding & $283 / 9 e-77$ \\
\hline UBQIO & TC203625 & AT4G05320 & Ubiquitin 10 & $\begin{array}{l}\text { Protein binding, protein } \\
\text { modification process }\end{array}$ & $734 / 0$ \\
\hline GmBFT & EF532597 & AT5G62040 & $\begin{array}{c}\text { Brother of FT and TFLI } \\
\text { protein }\end{array}$ & $\begin{array}{l}\text { Phosphatidylethanola - } \\
\text { mine binding }\end{array}$ & $23 I / 4 e-6 I$ \\
\hline
\end{tabular}

aAll soybean sequences except TUA were ESTs based on the Arabidopsis proteins determined via BLASTX. bClosest Arabidopsis homolog identified using Tair BLAST [50]. AGI protein database was queried with soybean nucleotide sequences using BLASTX. 
Table 6: Primers and amplicons for each of the 10 HKGs and GmBFT

\begin{tabular}{|c|c|c|c|c|c|c|}
\hline Name & Forward Primer Sequence [5'-3'] & Reverse Primer Sequence [5'-3'] & Amplicon Length (bp) & Primer location $^{\mathrm{a}}$ & $E(\%)$ & $\mathrm{R}^{2}$ \\
\hline ACTII & CGGTGGTTCTAT CTTGGCATC & GTCTTTCGCTTCAA TAACCCTA & 142 & $\mathrm{D}$ & 98.04 & 0.9979 \\
\hline ACT2/7 & СTTCССТCAGCA ССTTCCAA & GGTCCAGCTTTCA CACTCCAT & 119 & $\mathrm{D}$ & 109.56 & 0.9979 \\
\hline G6PD & ACTCCTTGATAC CGTTGTCCAT & GTTTGTTATCCGCC TACAGCCT & 126 & $\mathrm{D}$ & 98.59 & 0.9990 \\
\hline$E L F I B$ & GTTGAAAAGCCA GGGGACA & TCTTACCCCTTGA GCGTGG & 118 & $\mathrm{D}$ & 93.15 & 0.9883 \\
\hline UBC2 & ТССССТСАСАСС СТТССТС & CCATCCCAAGGGG TGTCAT & 155 & $\mathrm{D}$ & 100.60 & 0.9988 \\
\hline CYP2 & CGGGACCAGTGTGCTTCTTCA & ССССТССАСTACAAAGGCTCG & 154 & S & 82.31 & 0.9848 \\
\hline ELFIA & GACCTTCTTCGT TTCTCGCA & CGAACCTCTCAAT CACACGC & 195 & $\mathrm{D}$ & 98.21 & 0.9981 \\
\hline TUB & CCTCGTTCGAAT TCGCTTTTTG & CAACTGTCTTGTC GCTTGGCAT & $16 \mid$ & S & 100.84 & 0.9956 \\
\hline TUA & AGGTCGGAAACT CСTGCTGG & AAGGTGTTGAAGG CGTCGTG & 159 & S & 85.75 & 0.9829 \\
\hline UBQIO & CGCCTCTAATCT CGCAGTTCC & GTTGTCAATGGTG TCGGAGGA & 114 & $S$ & 120.28 & 0.9663 \\
\hline GmBFT & CCAAGGGAAATT GTGAGGTA & CTACTAAAAAGCC CCACAGC & 191 & $S$ & 101.65 & 0.9985 \\
\hline
\end{tabular}

${ }^{a} \mathrm{D}$, the two primers were located on different exons; $\mathrm{S}$, the two primers were located on the same exon.

\section{qRT-PCR}

qRT-PCR was conducted on ABI PRISM 7000 Sequence Detection System using power SYBR Green Mix (Applied Biosystems, USA). Each reaction was run in a $20 \mu \mathrm{l}$ volume which contained $8.8 \mu \mathrm{l}$ cDNA equal to $20 \mathrm{ng}, 10 \mu \mathrm{l} 2$ $\times$ power SYBR mix, $0.6 \mu$ l each primer to a final concentration of $300 \mathrm{nM}$. All the reactions were performed as the following conditions: $2 \mathrm{~min}$ at $50^{\circ} \mathrm{C}, 10 \mathrm{~min}$ at $95^{\circ} \mathrm{C}$, and 40 cycles of $10 \mathrm{~s}$ at $95^{\circ} \mathrm{C}$, and $1 \mathrm{~min}$ at $60^{\circ} \mathrm{C}$ in 96 -well optical reaction plates (Applied Biosystems, USA). To verify the specificity of the amplicon for each primer pair, a melting curve was made from $60^{\circ} \mathrm{C}$ to $95^{\circ} \mathrm{C}$ at the end of each PCR run and all the ten primer pairs amplified a single product. The PCR efficiencies showed in Table 6 for each gene was determined with the slope of a liner regression model. Each cDNA sample pool was bulked and then used as the PCR template in a range of 50, 25, 10, 5, and 2 ng [22]. The corresponding real-time PCR efficiencies were calculated according to the equation: $\mathrm{E}=10^{-1 / \text { slope }}$ [4].

\section{Data processing}

Expression levels of the ten HKGs in all the sample pools were determined by the number of cycles $(\mathrm{Ct})$ needed for the amplification related fluorescence to reach a specific threshold level of detection [39]. The raw $\mathrm{Ct}$ value obtained from ABI 7000 after each PCR run was converted into relative quantities using the PCR efficiencies for each gene according to the requirement of geNorm software $[11,49]$ to calculate gene expression stability $(\mathrm{M})$. The expression stability of the ten HKGs was also determined by the 'Stability index' [16] and $\Delta \mathrm{Ct}$ approach [15] to compare the three methods in all the 21 sample pools.

\section{Abbreviations}

HKG, housekeeping gene; qRT-PCR, quantitative realtime PCR; ACT11, actin11; ACT2/7, actin2/7; G6PD, glucose-6-phosphate dehydrogenase; ELF1B, eukaryotic elongation factor 1-beta; UBC2, ubiquitin-conjugating enzyme E2;
ELF1A, eukaryotic elongation factor 1-alpha; TUB, beta-tubulin; TUA, alpha-tubulin; CYP2, cyclophilin; UBQ10, ubiquitin 10; ZGGG, Zigongdongdou; HH27, Heihe No. 27; ZH24, Zhonghuang No. 24; SN14, Suinong No. 14; SD, short day; LD, long day; StdDev, standard deviation; CV, coefficient variation; GmBFT, Glycine max brother of FT and TFL1.

\section{Authors' contributions}

BJ performed all the experimental procedures, data analysis, draft the manuscript and was the primary author of the manuscript. BL participated in data analysis, tables and figures drawing and manuscript revising. YB designed the study. WH participated in the experimental process and provided technical support throughout the experimental process. CW performed the sample preparation. TH supervised the study, revised the manuscript critically and gave financial support to the study.

\section{Additional material}

\section{Additional File 1}

HKG comparisons. Mean $\triangle C$ t values were given for the mean difference between the genes over the 21 sample pools. SteDev was given for the variation in Ct values over the 21 sample pools.

Click here for file

[http://www.biomedcentral.com/content/supplementary/14712199-9-59-S1.xls]

\section{Acknowledgements}

We thank Hongbo Sun and Shikui Song for suggestions in performing the experiment and Jiantian Leng and Ying Wang for assistance in data analysis. We especially thank Charles $\mathrm{H}$. Leseberg and Tore Brembu for reading the manuscript and their suggestions for the writing process. This work was supported by the National Natural Science Foundation of China (3047 I054), National High Technology Research and Development Program of China (2007AA I OZI33) and Beijing Natural Science Foundation (5042019). 


\section{References}

I. Bustin S: Absolute quantification of mRNA using real-time reverse transcription polymerase chain reaction assays. Journal of molecular endocrinology 2000, 25:169-193.

2. Ginzinger DG: Gene quantification using real-time quantitative PCR: an emerging technology hits the mainstream Experimental hematology 2002, 30(6):503-5I2

3. Garson JA, Grant PR, Ayliffe U, Ferns RB, Tedder RS: Real-time PCR quantitation of hepatitis $B$ virus DNA using automated sample preparation and murine cytomegalovirus internal control. Journal of virological methods 2005, I 26( I-2):207-2I3.

4. Radonic A, Thulke S, Mackay IM, Landt O, Siegert W, Nitsche A: Guideline to reference gene selection for quantitative realtime PCR. Biochemical and biophysical research communications 2004 , 3 I 3(4):856-862.

5. Gal AB, Carnwath JW, Dinnyes A, Herrmann D, Niemann H, Wrenzycki $\mathrm{C}$ : Comparison of real-time polymerase chain reaction and end-point polymerase chain reaction for the analysis of gene expression in preimplantation embryos. Reproduction, fertility, and development 2006, I8(3):365-37I.

6. Jung $M$, Ramankulov A, Roigas J, Johannsen M, Ringsdorf M, Kristiansen $G$, Jung $K$ : In search of suitable reference genes for gene expression studies of human renal cell carcinoma by realtime PCR. BMC molecular biology 2007, 8:47.

7. de Kok JB, Roelofs RW, Giesendorf BA, Pennings JL, Waas ET, Feuth T, Swinkels DW, Span PN: Normalization of gene expression measurements in tumor tissues: comparison of 13 endog. enous control genes. Laboratory investigation; a journal of technica methods and pathology 2005, 85(I): I54-159.

8. Thellin O, Zorzi W, Lakaye B, De Borman B, Coumans B, Hennen G, Grisar T, Igout A, Heinen E: Housekeeping genes as internal standards: use and limits. Journal of biotechnology 1999 75(2-3):291-295

9. Schmittgen TD, Zakrajsek BA: Effect of experimental treatment on housekeeping gene expression: validation by real-time, quantitative RT-PCR. Journal of biochemical and biophysical methods 2000, 46(I-2):69-8I.

10. Tricarico C, Pinzani P, Bianchi S, Paglierani M, Distante V, Pazzagli M, Bustin SA, Orlando C: Quantitative real-time reverse transcription polymerase chain reaction: normalization to rRNA or single housekeeping genes is inappropriate for human tissue biopsies. Analytical biochemistry 2002, 309(2):293-300.

II. Vandesompele J, De Preter K, Pattyn F, Poppe B, Van Roy N, De Paepe A, Speleman F: Accurate normalization of real-time quantitative RT-PCR data by geometric averaging of multiple internal control genes. Genome biology 2002 3(7):RESEARCH0034.

12. Singh R, Green MR: Sequence-specific binding of transfer RNA by glyceraldehyde-3-phosphate dehydrogenase. Science 1993, 259(5093):365-368.

13. Ishitani R, Sunaga K, Hirano A, Saunders P, Katsube N, Chuang DM: Evidence that glyceraldehyde-3-phosphate dehydrogenase is involved in age-induced apoptosis in mature cerebellar neurons in culture. Journal of neurochemistry 1996, 66(3):928-935.

14. Andersen CL, Jensen JL, Orntoft TF: Normalization of real-time quantitative reverse transcription-PCR data: a model-based variance estimation approach to identify genes suited for normalization, applied to bladder and colon cancer data sets. Cancer research 2004, 64(I 5):5245-5250.

15. Silver N, Best S, Jiang J, Thein SL: Selection of housekeeping genes for gene expression studies in human reticulocytes using real-time PCR. BMC molecular biology 2006, 7:33.

16. Brunner AM, Yakovlev IA, Strauss SH: Validating internal controls for quantitative plant gene expression studies. $B M C$ plant biology 2004, 4: I4.

17. Goossens K, Van Poucke M, Van Soom A, Vandesompele J, Van Zeveren A, Peelman LJ: Selection of reference genes for quantitative real-time $P C R$ in bovine preimplantation embryos. $B M C$ developmental biology 2005, 5:27.

18. Ingerslev HC, Pettersen EF, Jakobsen RA, Petersen CB, Wergeland $H I$ : Expression profiling and validation of reference gene candidates in immune relevant tissues and cells from Atlantic salmon (Salmo salar L.). Molecular immunology 2006, 43(8): I| $194-1201$.

19. Ding J, Jia J, Yang L, Wen H, Zhang C, Liu W, Zhang D: Validation of a rice specific gene, sucrose phosphate synthase, used as the endogenous reference gene for qualitative and real-time quantitative PCR detection of transgenes. Journal of agricultural and food chemistry 2004, 52(I I ):3372-3377.

20. Jain M, Nijhawan A, Tyagi AK, Khurana JP: Validation of housekeeping genes as internal control for studying gene expression in rice by quantitative real-time PCR. Biochemical and biophysical research communications 2006, 345(2):646-65I.

21. Kim BR, Nam HY, Kim SU, Kim SI, Chang YJ: Normalization of reverse transcription quantitative-PCR with housekeeping genes in rice. Biotechnology letters 2003, 25(2I): |869-1872.

22. Nicot N, Hausman JF, Hoffmann L, Evers D: Housekeeping gene selection for real-time RT-PCR normalization in potato during biotic and abiotic stress. Journal of experimental botany 2005 , 56(42I):2907-2914.

23. Czechowski T, Stitt M, Altmann T, Udvardi MK, Scheible WR Genome-wide identification and testing of superior reference genes for transcript normalization in Arabidopsis. Plant physiology 2005, I39(I):5-17.

24. Garner $W$, Alland $H$ : Effect of the relative length of day and night and other factors of the environment on growth and reproduction in plants. J Agric Res 1920, I 8:553-606.

25. Borthwick H, Parker $M$ : Influence of photoperiods upon the differentiation of meristems and the blossoming of Biloxi soybeans. Bot Gaz 1938, 99:825-839.

26. Munir J, Dorn LA, Donohue K, Schmitt J: The effect of maternal photoperiod on seasonal dormancy in Arabidopsis thaliana (Brassicaceae). Am J Bot 200I, 88(7): I 240-I249.

27. Han T, Wu C, Tong Z, Mentreddy RS, Tan K, Gai J: Postflowering photoperiod regulates vegetative growth and reproductive development of soybean. Enviromental and Experimental Botany 2006, 55:120-129.

28. Wu C, Ma Q, Yam KM, Cheung MY, Xu Y, Han T, Lam HM, Chong $\mathrm{K}$ : In situ expression of the $\mathrm{GmNMH7}$ gene is photoperioddependent in a unique soybean (Glycine max [L.] Merr.) flowering reversion system. Planta 2006, 223(4):725-735.

29. Masuda J, Urakawa T, Ozaki Y, Okubo H: Short photoperiod induces dormancy in Lotus (Nelumbo nucifera). Annals of botany 2006, 97(I):39-45.

30. Kantolic AG, Slafer GA: Photoperiod sensitivity after flowering and seed number determination in indeterminate soybean cultivars. Field Crops Res 2001, 72:109-II8.

31. Kantolic AG, Slafer GA: Development and seed number in indeterminate soybean as affected by timing and duration of exposure to long photoperiods after flowering. Annals of botany 2007, 99(5):925-933.

32. Putterill J, Robson F, Lee K, Simon R, Coupland G: The CONSTANS gene of Arabidopsis promotes flowering and encodes a protein showing similarities to zinc finger transcription factors. Cell 1995, 80(6):847-857.

33. Teper-Bamnolker $P$, Samach A: The flowering integrator $F T$ regulates SEPALLATA3 and FRUITFULL accumulation in Arabidopsis leaves. Plant Cell 2005, I 7(I 0):266I-2675.

34. Corbesier L, Vincent C, Jang S, Fornara F, Fan Q, Searle I, Giakountis A, Farrona S, Gissot L, Turnbull C, Coupland G: FT protein movement contributes to long-distance signaling in floral induction of Arabidopsis. Science 2007, 3 I 6(5827): I030-1033.

35. Jung JH, Seo YH, Seo PJ, Reyes JL, Yun J, Chua NH, Park CM: The GIGANTEA-Regulated MicroRNAI72 Mediates Photoperiodic Flowering Independent of CONSTANS in Arabidopsis. Plant Cell 2007, I9(9):2736-2748.

36. Toegel S, Huang W, Piana C, Unger FM, Wirth M, Goldring MB Gabor $F$, Viernstein $H$ : Selection of reliable reference genes for qPCR studies on chondroprotective action. BMC molecular biology 2007, 8: 13 .

37. Sun H, Liu $Y$, Hu P, Hou W, Wu C, Cao D, Han T: Cloning and characterization of GmBFT, a soybean BFT homologue encoding the phosphatidylethanolamine-binding protein. DNA Sequcence 2008. (accepted)

38. Kobayashi $\mathrm{Y}$, Kaya H, Goto K, Iwabuchi M, Araki T: A pair of related genes with antagonistic roles in mediating flowering signals. Science 1999, 286(5446): 1960-1962.

39. Walker NJ: Tech. Sight. A technique whose time has come. Science 2002, 296(5567):557-559.

40. Ohl F, Jung M, Xu C, Stephan C, Rabien A, Burkhardt M, Nitsche A, Kristiansen G, Loening SA, Radonic A, Jung K: Gene expression studies in prostate cancer tissue: which reference gene 
should be selected for normalization? Journal of molecular medicine (Berlin, Germany) 2005, 83(12): I0I4-I024.

41. Steinau M, Rajeevan MS, Unger ER: DNA and RNA references for qRT-PCR assays in exfoliated cervical cells. J Mol Diagn 2006, 8(I): I|3-II8.

42. Reid KE, Olsson N, Schlosser J, Peng F, Lund ST: An optimized grapevine RNA isolation procedure and statistical determination of reference genes for real-time RT-PCR during berry development. BMC plant biology 2006, 6:27.

43. Hochstrasser M: Evolution and function of ubiquitin-like protein-conjugation systems. Nature cell biology 2000, 2(8):EI 53-I 57.

44. Filby AL, Tyler CR: Appropriate 'housekeeping' genes for use in expression profiling the effects of environmental estrogens in fish. BMC molecular biology 2007, 8: 10 .

45. Ohl F, Jung M, Radonic A, Sachs M, Loening SA, Jung K: Identification and validation of suitable endogenous reference genes for gene expression studies of human bladder cancer. The Journal of urology 2006, I75(5): 1915-1920.

46. Han T, Wang J: Studies on the post-flowering photoperiodic responses in soybean. Acta Botanica Sinica 1995, 37( I I):863-869.

47. Soybean EST database [http://compbio.dfci.harvard.edu/tgi/cgibin/tgi/gimain.pl?gudb=soybean]

48. Premierbiosoft [http://www.premierbiosoft.com/faq.html]

49. geNorm [http://medgen.ugent.be/ - jvdesomp/genorm/]

50. TAIR BLAST [http://www.arabidopsis.org/Blast/]

\section{Publish with Bio Med Central and every scientist can read your work free of charge}

"BioMed Central will be the most significant development for disseminating the results of biomedical research in our lifetime. "

Sir Paul Nurse, Cancer Research UK

Your research papers will be:

- available free of charge to the entire biomedical community

- peer reviewed and published immediately upon acceptance

- cited in PubMed and archived on PubMed Central

- yours - you keep the copyright

Submit your manuscript here:

http://www.biomedcentral.com/info/publishing_adv.asp 